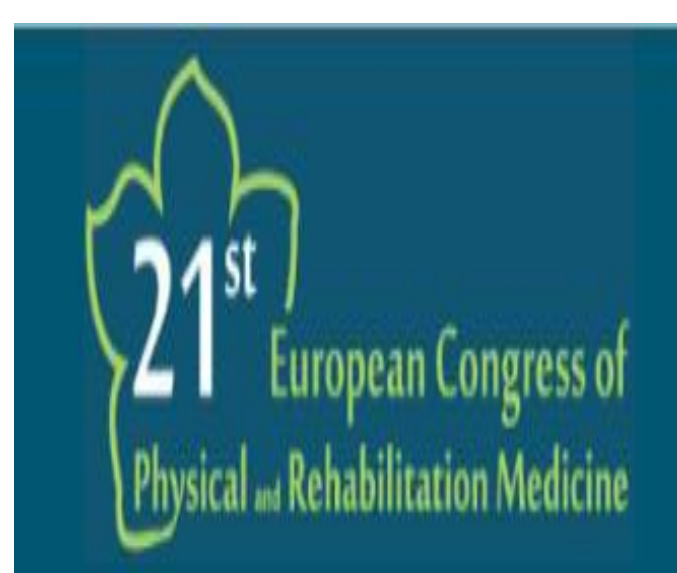

\title{
PATELLOFEMORAL PAIN (PFP): LITERATURE REVISION
}

Miguel Joana ${ }^{1}$, Diana Mesquita ${ }^{1}$, Inês Natário ${ }^{1}$, João Carvalho ${ }^{1}$, José Carvalho ${ }^{1}$, Edgar Tamegão ${ }^{1}$, Sofia Amorim ${ }^{1}$, Gonçalo Borges ${ }^{1}$

${ }^{1}$ Physical Medicine and Rehabilitation, Hospital da Prelada, Serviço de Medicina Física e Reabilitação Porto, Portugal

\section{Introduction}

The patellofemoral pain (PFP) is a common finding in clinical practice that presents as a insidious and diffuse pain in the anterior face of the knee usually in activities of squats stairs, running and prolonged periods in the seated position. It is commonly associated with decreased sports performance, limiting physical and daily life activities, often evolving to chronicity. Physical and pharmacological agents and some kinesiological techniques have been applied inconsistently.

\section{Purpose}
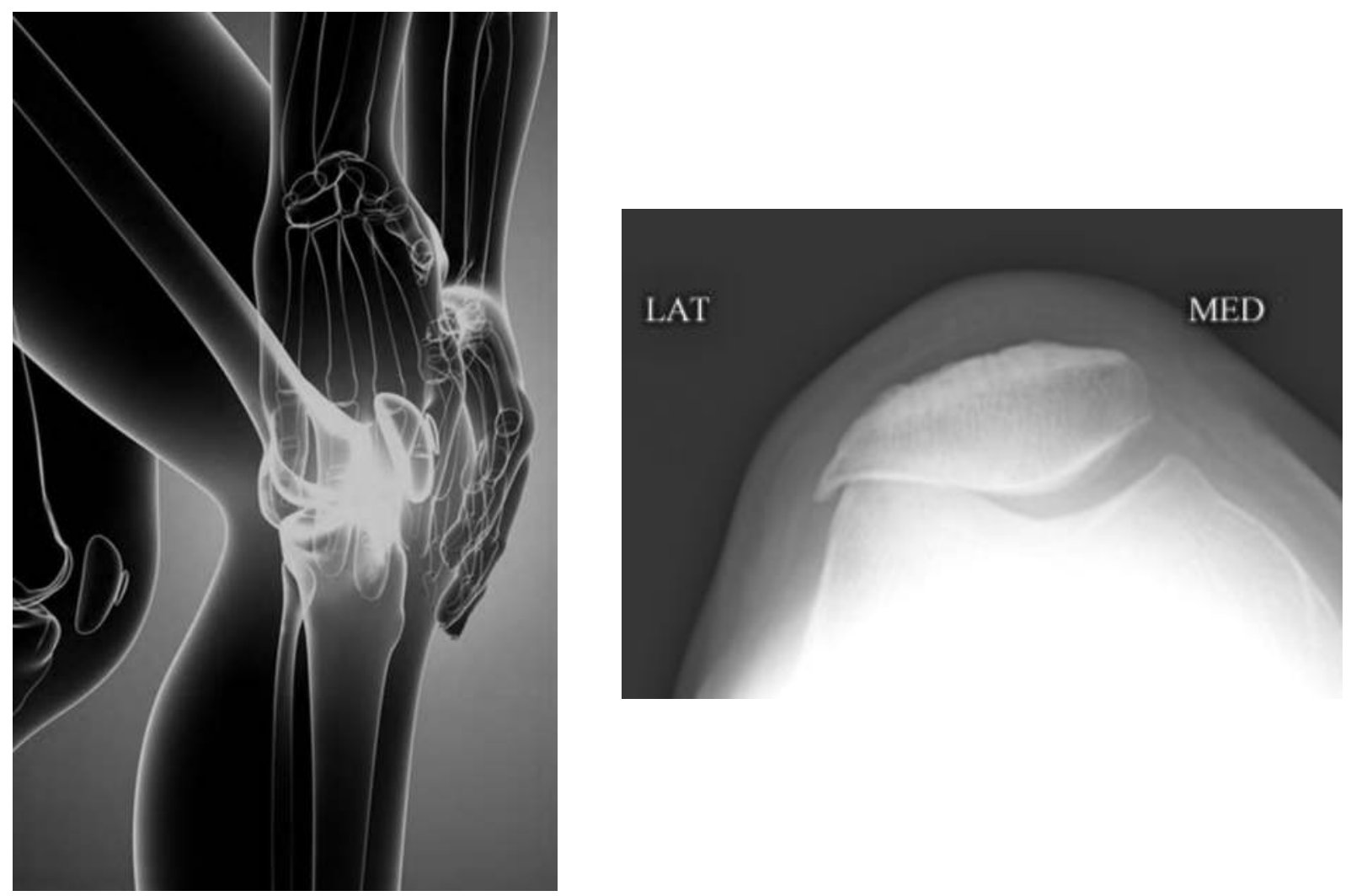

Through a review of the recently published relevant literature, namely "2017 Patellofemoral pain consensus statement from the 5th International Patellofemoral Pain Research Retreat" occurred in July 2017, I intend to transmit the new knowledge about pathophysiology, diagnosis and conservative treatment in this pathology.

\section{Method}

Search in the Pubmed electronic database (from January 1999 to July 2017), using as keywords: "Patellofemoral pain or syndrome" and "runner's knee".

\section{Results}

The latest international consensus demonstrates that the isolated prescription of electrophysical agents, the mobilizations of the patella, knee and lower back are no longer recommended. Interventions such as acupuncture have a low level of evidence, and their benefit is uncertain and therefore not recommended. The effective intervention of Physical and Rehabilitation Medicine (PRM) involves muscular strengthening focused on the hip and knee muscles, tapping, orthotic placement and the combined application of physical agents, constituting the first line of action.
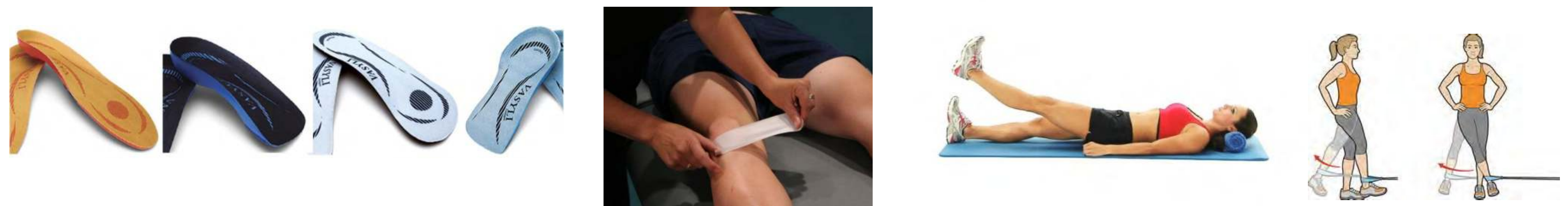

\section{Conclusion:}

Physical and Rehabilitation Medicine presents a major role in PFP, given that the first-line treatment is conservative. It is stimulating for us, while PRM doctors have the possibility to make a difference in a so common pathology. It is essential the knowledge of the new therapeutic indications and how they should be used to make our intervention more successful. 